\title{
Accessory right hepatic artery and aberrant bile duct in the hepatocystic triangle: a rare case with clinical implications
}

\author{
N. Eid'10, M. Allouh1', Y. Ito², K. Taniguchi², E. Adeghate ${ }^{1}$ \\ ${ }^{1}$ Department of Anatomy, College of Medicine and Health Sciences, United Arab Emirates University, \\ Al Ain, United Arab Emirates \\ 2Department of General and Gastroenterological Surgery, Osaka Medical and Pharmaceutical University, \\ Takatsuki, Osaka, Japan
}

[Received: 10 May 2021; Accepted: 12 June 2021; Early publication date: 29 June 2021]

\begin{abstract}
Awareness of variations in the hepatic vasculature and biliary system is extremely important for avoiding iatrogenic injury in upper-abdominal surgery. The objective of this study is to describe a rare case of abnormal vascular and biliary structures in the hepatocystic triangle (HCT) (the modern Calot's triangle). During anatomical dissection of the coeliac trunk (CT) in an old man, the authors observed the presence of a hepatosplenic trunk arising from the CT and bifurcating into common hepatic and splenic arteries. The common hepatic artery divided into hepatic artery proper and gastroduodenal artery. The presence of accessory right hepatic artery (ARHA) arising from the superior mesenteric artery was also notable. The aberrant artery ascended retropancreatically ventral to the splenic vein, then posterolaterally to the portal vein before termination into the right hepatic lobe in the HCT. Within this triangle, there was an aberrant bile duct originating in the right hepatic lobe and ending in the common hepatic duct. This accessory duct crossed the ARHA and an associated branch (the cystic artery). There is no known previous report on the co-existence of an ARHA and an aberrant bile duct within the HCT, in addition to the hepatosplenic trunk. The clinical implications of the current case are addressed in discussion. (Folia Morphol 2022; 81, 3: 798-803)
\end{abstract}

Key words: hepatocystic triangle, triangle of Calot, accessory right hepatic artery, aberrant bile duct, hepatosplenic trunk, variations, coeliac trunk

\section{INTRODUCTION}

Variations in blood supply to the hepatic arteries are not rare. According to anatomy text books, the common hepatic branch of the coeliac trunk (CT) supplies the liver in $55-88 \%$ of cases. Variations of liver blood supply are frequent. Thus, the liver may be supplied by aberrant branches of the left gastric artery, the superior mesenteric artery (SMA), the aorta or other gut arteries. These aberrant arteries may be accessory or replaced $[4,5,6,9,22]$.

Recognition of variations in hepatic arterial anatomy is of major importance in the planning and conducting of surgical and radiological procedures on the upper-abdomen area, such as transplantation, cholecystectomy and pancreaticoduodenectomy $[6,8,9,22]$. Based on records of 1000 patients who

Address for correspondence: Dr. N. Eid, Department of Anatomy, College of Medicine and Health Sciences, United Arab Emirates University, P.O. Box 17666, Al Ain, United Arab Emirates, tel: +971568088037, e-mails: nabileidm@uaeu.ac.ae; nabileidm2@gmail.com

This article is available in open access under Creative Common Attribution-Non-Commercial-No Derivatives 4.0 International (CC BY-NC-ND 4.0) license, allowing to download articles and share them with others as long as they credit the authors and the publisher, but without permission to change them in any way or use them commercially. 


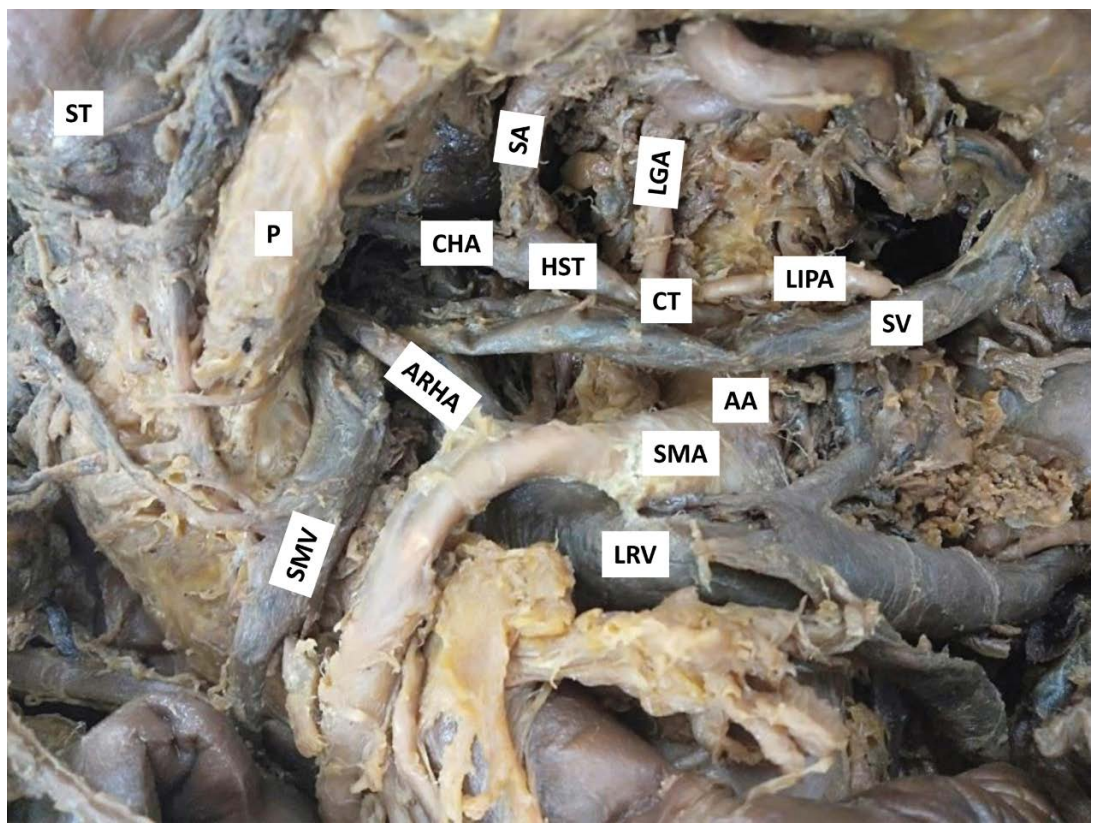

Figure 1. Accessory right hepatic artery (ARHA) arising from the superior mesenteric artery (SMA) in the pancreatic bed. The stomach (ST) and pancreas $(\mathrm{P})$ are retracted to show the variation; AA — abdominal aorta; CHA — common hepatic artery; CT — coeliac trunk; HST — hepatosplenic trunk; LGA — left gastric artery; LIPA — left inferior phrenic artery; LRV — left renal vein; SA — splenic artery; SMA — superior mesenteric artery; SMV — superior mesenteric vein; SV — splenic vein.

underwent liver harvesting for orthotopic transplantation, Hiatt et al. [9] investigated the surgical anatomy of hepatic arteries. They found six types of hepatic artery variations. Type $1-$ normal (the common hepatic artery [CHA] branched from CT) (75.7\%); type 2 - replaced (accessory) left hepatic artery branched from left gastric (9.7\%); type 3 - replaced (accessory) right hepatic artery branched from SMA (10.6\%); type 4 - replaced or accessory right hepatic artery + replaced or accessory left hepatic artery (2.3\%); type 5 - the CHA branched from SMA (1.5\%), and type 6 - the CHA originated from abdominal aorta $(0.2 \%)$. The presence of an aberrant right hepatic artery is the most frequently encountered variation, and this may be either a replacement or an accessory (Hiatt type III, $10.6 \%$ incidence) [5, 9]. This aberrant artery may be accidentally injured during upper-abdomen operations resulting in fatal complications such as severe haemorrhage and hepatic necrosis $[5,6,9,22]$. Importantly, biliary tract variations - specifically, the presence of aberrant or accessory bile ducts - may complicate laparoscopic cholecystectomy. During this procedure, only two structures (the cystic duct and the cystic artery) should usually be seen entering the gallbladder within the hepatocystic triangle (HCT) (the modern Calot's triangle), which is bounded by the cystic duct, the common hepatic duct (CHD) and the liver. This is different from the earlier description of Calot: the Calot's triangle is demarcated by the cystic duct on right side, the CHD on left side, and the cystic artery above $[1,16,17]$. In a previous study, the authors reported a rare case of a right hepatic artery forming a caterpillar hump perforating Calot's triangle into the cystic plate in association with a variant branching pattern of the CT [7]. In the current study, the authors referenced cadaveric dissection results to examine another rare case involving the coexistence of three variations with clinically important implications in hepatobiliary and pancreatic surgery. These variations include two structures (an accessory right hepatic artery [ARHA] and an aberrant bile duct) within the HCT, in addition to the hepatosplenic trunk.

\section{CASE REPORT}

During anatomical dissection of the $\mathrm{CT}$ in a male cadaver (among 8 dissections) at the Department of Anatomy in the College of Medicine and Health Sciences at the United Arab Emirates University, multiple variations in hepatic vasculature and the biliary system were observed. The CT was exposed after severing the greater omentum and upward reflection of stomach. The CT bifurcated into the left gastric artery and hepatosplenic trunk (Fig. 1). The hepatosplenic trunk divided into CHA and splenic artery. 


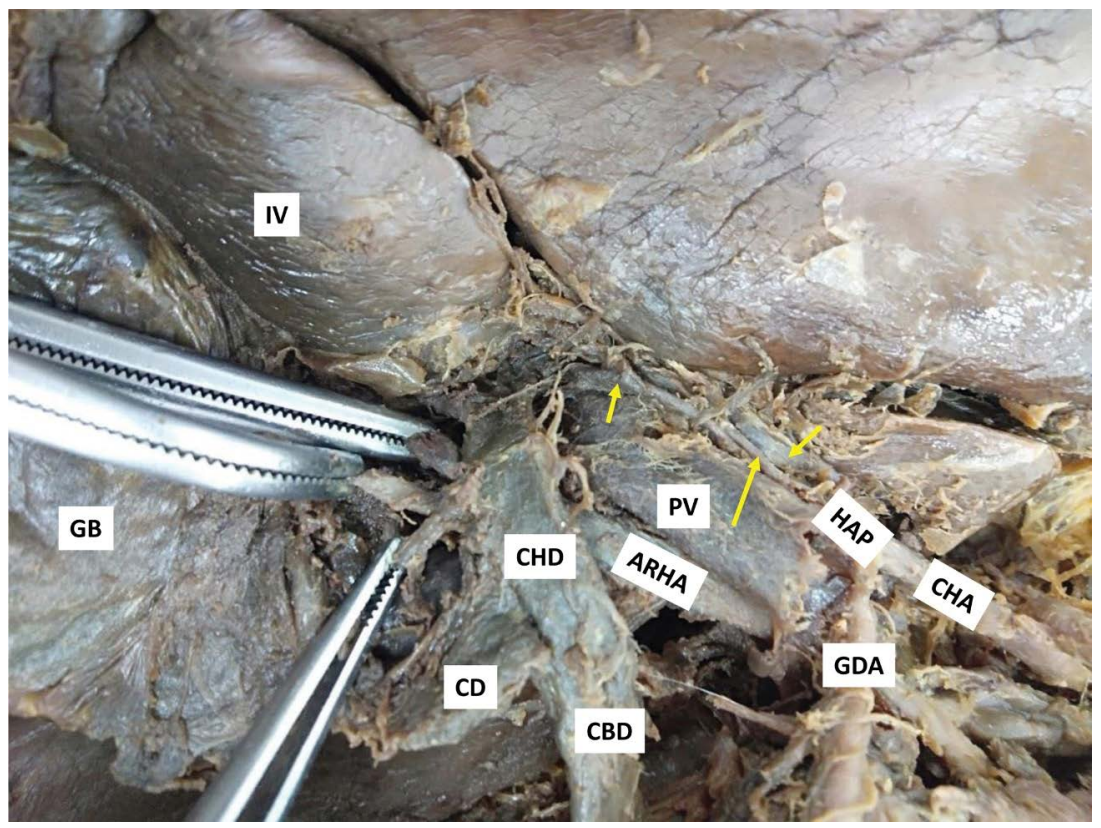

Figure 2. Accessory right hepatic artery (ARHA) passing into the hepatocystic triangle in association with the accessory bile duct and cystic artery. The upper, middle and lower haemostats within the triangle hold the ARHA, cystic artery and anomalous bile duct, respectively. The yellow arrows mark the right, left and middle hepatic branches of the hepatic artery proper (HAP); CBD - common bile duct; $C D$ - cystic duct; CHA — common hepatic artery; CHD — common hepatic duct; GB — gallbladder; GDA — gastroduodenal artery; IV — hepatic segment 4; PV — portal vein.

The CHA then branched into gastroduodenal artery and hepatic artery proper (HAP), which trifurcated into right, left and middle hepatic branches (Fig. 2). This trifurcation of HAP was observed after dissection of the hepatoduodenal ligament. Further dissection of the pancreatic bed demonstrated the presence of an ARHA arising from the SMA. The ARHA ascended retropancreatically ventral to the splenic vein at its junction with the superior mesenteric vein parallel to the CHA (Fig. 1). Notably, it passed posterolaterally to the portal vein and the CHD, entering the HCT and terminating into the right hepatic lobe close to the cystic plate. In this triangle, the ARHA gave rise to the cystic artery, and both arteries were crossed ventrally by an aberrant bile duct arising from the right hepatic lobe and joining the right side of the CHD (Fig. 2). The gallbladder was dissected from the cystic plate via upward retraction and severing of its peritoneal attachment, thereby clearly revealing the contents of HCT - the ARHA, the aberrant bile duct and the cystic artery based on the criteria of Critical View of Safety (CVS) for laparoscopic cholecystectomy by Strasberg (Fig. 3) [16, 17]. The CVS has three requirements. First, the HCT must be cleared of fat and fibrous tissue. The second requirement is that the inferior part of the gallbladder be separated from the cystic plate (hepatic bed of gallbladder), the flat fibrous surface to which the non-peritonealised side of the gallbladder is attached. The third requirement is that only two structures (cystic duct and cystic artery), must be seen getting into the gallbladder. Once these three criteria have been fulfilled, CVS has been attained. The normal and variant anatomy of HCT is shown in Figure 4 with digital art based on schema of Figure 2 .

\section{DISCUSSION}

In the case reported here, the ARHA and the anomalous bile duct were observed simultaneously as variant structures in the $\mathrm{HCT}$, along with a variant branching pattern of the $\mathrm{CT}$ with formation of the hepatosplenic trunk. Up to our knowledge, there are no known previous reports on the coexistence of these variations.

The detection of an ARHA arising from the SMA in the current case is consistent with Hiatt type III (10.6\% incidence) $[5,9]$. This artery ascended superficial to the splenic vein and deep to the pancreatic neck. Accordingly, there may have been compression of the splenic vein by the enlarged ARHA and the pancreas, resulting in splenic vein thrombosis and left-sided portal hypertension [19]. In addition, the entry of the 


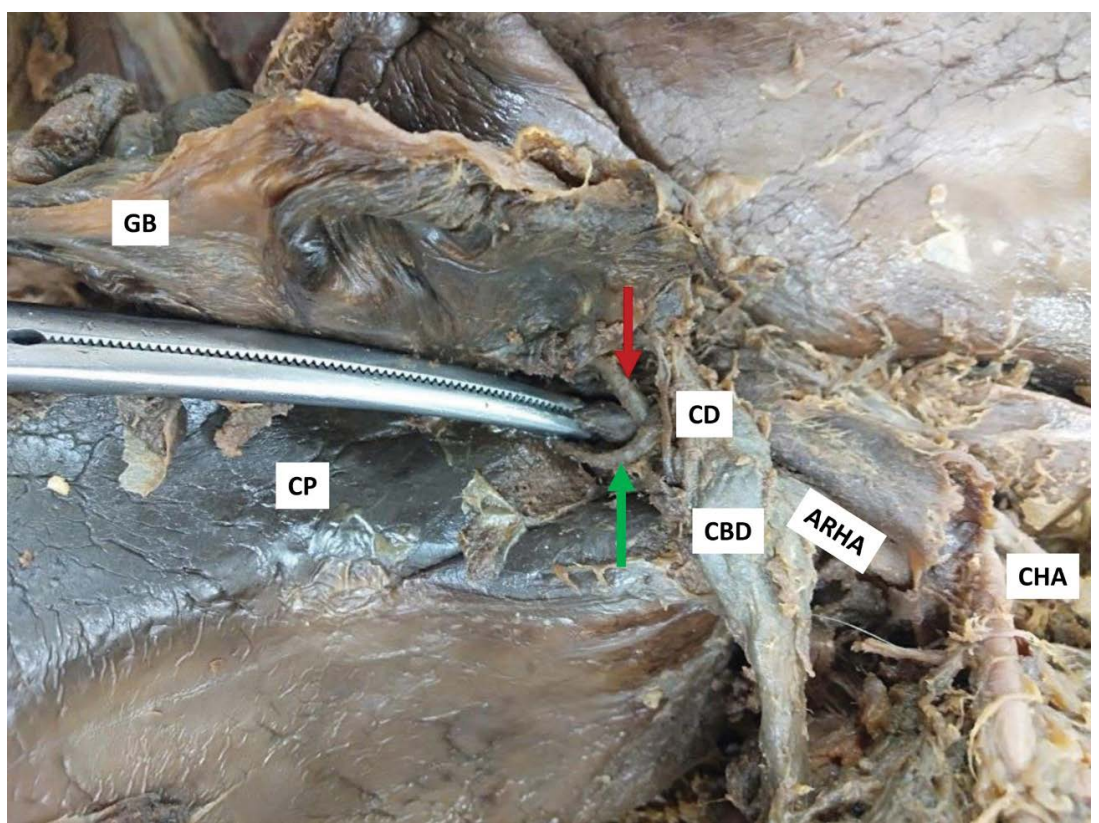

Figure 3. Dissection and upward retraction of gall bladder (GB) for exposure of cystic plate and detection of anomalous structures in the hepatocystic triangle based on criteria of Critical View of Safety. Note that the accessory right hepatic artery (ARHA) is grasped by the haemostat, whereas the cystic artery and aberrant bile duct are marked by the red and green arrows, respectively; CBD — common bile duct; $\mathrm{CD}$ - cystic duct; $\mathrm{CHA}$ — common hepatic artery; $\mathrm{CP}$ — cystic plate.
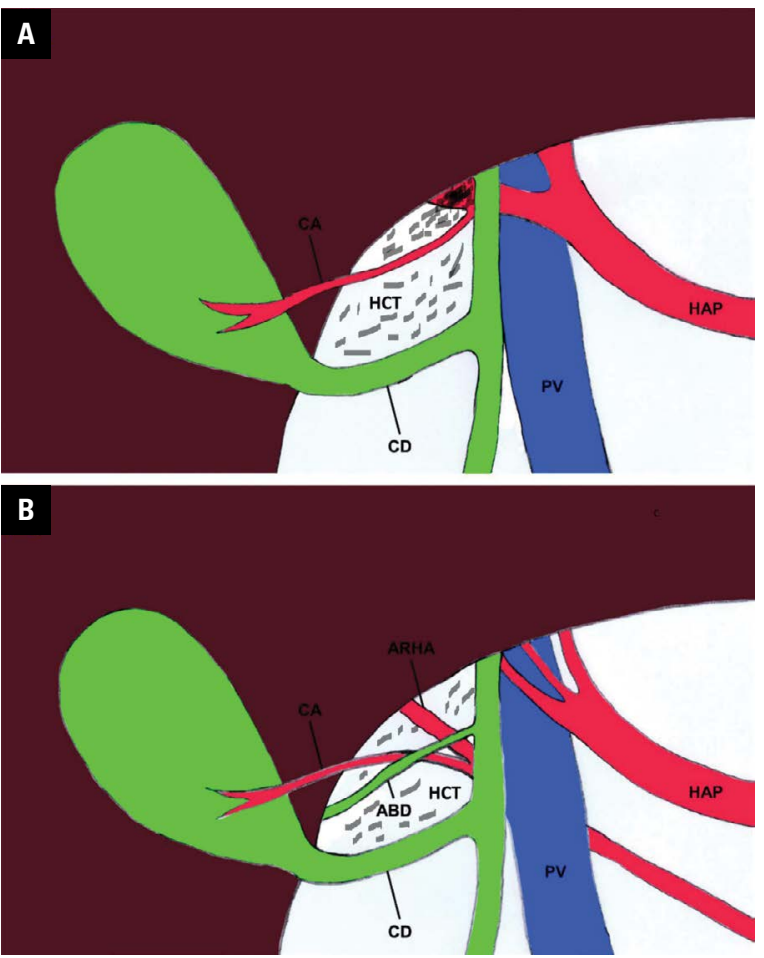

Figure 4. A schema and digital art (A, B) showing the normal (A) and variant anatomy of the hepatocystic triangle (HCT; highlighted with black lines) of Calot (B). The digital art of $B$ is based on Figure 2; $\mathrm{ABD}$ - aberrant bile duct; ARHA — accessory right hepatic artery; $\mathrm{CA}$ - cystic artery; $\mathrm{CD}$ - cystic duct; HAP — hepatic artery proper; PV — portal vein.
ARHA into the HCT has great surgical relevance, as injury may occur during laparoscopic cholecystectomy with catastrophic complications such as bleeding and liver ischaemia $[6,16,17,22]$. The ARHA may also be damaged in various procedures associated with tumour-related pancreaticoduodenectomy [8] . Developmentally, the presence of ARHA may result from persistence and/or abnormal regression of the embryonic hepatic arteries [5].

The presence of an ARHA within the HCT has been reported in a couple of studies $[6,12,15]$, but was here associated with the aberrant bile duct, which may complicate CVS during laparoscopic cholecystectomy $[16,17]$. On the other side, some studies have shown that the replaced right hepatic artery is a beneficial variant in the right hepatic lobe living-donor transplant. This could be explained by the fact that the shorter hepatic artery graft could lead to a postoperative complication (hepatic artery thrombosis). However, the replaced right liver artery in such cases provides a longer and bigger graft, thereby reducing the chances of hepatic thrombosis. This could be applicable to our case as ARHA seems to be longer than the HAP [22]. Recent studies investigated the presence of ARHA using computed tomography, multidetector computed tomography 
angiography and magnetic resonance imaging (MRI). An enhanced computed tomography scan revealed variations in hepatic arteries in a 67-year-old woman diagnosed with intrahepatic cholangiocarcinoma [11]. These variations included ARHA branching from SMA and an accessory left hepatic artery arising from the left gastric artery. Using computed tomography angiography or/and MRI angiography findings in 532 patients, Yamaguchi et al. [21] observed a case of aberrant right hepatic artery arising from the gastroduodenal artery. They recommended the use of preoperative computed tomography and/or MRI and intraoperative liver Doppler ultrasound for the prevention of injury during pancreaticoduodenectomy in patients with this type of hepatic artery variation. Some researchers believe that ultrasound is always the method of choice for the diagnosis of various hepatobiliary diseases. They recommend a perfect study and a good understanding of the sonographic appearance of the normal hepatic vascular tree and its anatomical variations [3].

Importantly, the laparoscopic cholecystectomy CVS requirements $[16,17]$ applied during HCT dissection in the current study can also be used for practical anatomy teaching in medical schools. This may encourage undergraduate and postgraduate students to perform research on anatomical variations with clinical relevance.

Accidental injury or ligation of an aberrant bile duct as observed in the present case during cholecystectomy may result in severe complications such as biliary leakage and biliary cirrhosis $[10,13,16-18]$. However, some researchers consider any bile duct activity from the right lobe of the liver and interaction with the $\mathrm{CHD}$ as aberrant. Therefore, the lack of interductal communication in the liver may indicate that this duct may be the sole drainage of this particular part of the liver. When such an aberrant duct enters the main duct near the cystic duct, it may be easily injured, resulting in partial or total segmental duct occlusion. These ducts have a relatively high prevalence $(8.4 \%)$ and should be investigated using intraoperative cholangiography [10]. As shown in Figure 4, the cystic artery in our case is bridged by the aberrant bile duct, therefore, there is great possibility of injury of the duct during cholecystectomy. A recent study for the first time reported that fluorescence cholangiography concomitant with angiography was useful for identifying an aberrant bile duct and the cystic artery during laparoscopic cholecystectomy in old patient. The authors concluded that abnormal bile ducts are clinically important due to higher sensitivity to lesions during cholecystectomy. About $27 \%$ of clinically significant bile duct leakage was reported to be due to an aberrant bile duct lesion [18].

A recent report highlighted the coexistence of an ARHA and a left hepatic artery originating directly from the $C T$ in a patient who had undergone an elective pancreaticoduodenectomy via the Whipple procedure [2]. In the current case, in addition to the ARHA, the authors observed the presence of a hepatosplenic trunk arising from the CT. The presence of this trunk was reported as a type-II CT based on Adachi classification (4.3\%) [5]. Recent studies also found that a hepatosplenic trunk is observed in $10 \%$ of cases based on cadaveric dissection [14] and in $3.88 \%$ of subjects based on multidetector computed tomography [20]. The authors concluded that this CT variation was relevant to the planning of radiological and surgical procedures on the abdominal part of the oesophagus, the stomach, the duodenum, the liver, the pancreas, the gallbladder and the spleen, and in operations such as liver transplantation.

\section{CONCLUSIONS}

This unique study on the simultaneous presence of an ARHA, aberrant bile duct and a hepatosplenic trunk has important surgical implications; specifically, in hepatobiliary surgery. Further research on large number of cases using cadaveric dissection and imaging tools such as ultrasound, MRI and computed tomography are required to explore the abnormal contents of HCT.

\section{Acknowledgements}

We thank Mr. Anura J. Wanniarachchilage at Anatomy Department for help in dissection work.

Conflict of interest: None declared

\section{REFERENCES}

1. Abdalla S, Pierre S, Ellis H. Calot's triangle. Clin Anat. 2013; 26(4): 493-501, doi: 10.1002/ca.22170, indexed in Pubmed: 23519829.

2. Asif M, Anewenah LS, Reddy N, et al. Replaced right and left hepatic arteries: a variation in origin and course. BMJ Case Rep. 2017; 2017, doi: 10.1136/bcr-2016-218345, indexed in Pubmed: 28143859.

3. Battaglia S, Fachinetti C, Draghi F, et al. Ultrasound examination of the liver: Variations in the vascular anatomy. J Ultrasound. 2010; 13(2): 49-56, doi: 10.1016/j. jus.2010.07.003, indexed in Pubmed: 23396863. 
4. Bergamaschi R, Ignjatovic D. More than two structures in Calot's triangle. A postmortem study. Surg Endosc. 2000; 14(4): 354-357, doi: 10.1007/s004640000154, indexed in Pubmed: 10790554.

5. Chen H, Yano R, Emura S, et al. Anatomic variation of the celiac trunk with special reference to hepatic artery patterns. Ann Anat. 2009; 191(4): 399-407, doi: 10.1016/j. aanat.2009.05.002, indexed in Pubmed: 19540742.

6. Dolenšek J. Triple arterial blood supply to the liver and double cystic arteries. Folia Morphol. 2017; 76(3): 523-526, doi: 10.5603/FM.a2017.0008, indexed in Pubmed: 28150275.

7. Eid N, Ito $Y$, Otsuki Y. Right hepatic artery forming caterpillar hump, perforating Calot's triangle into the cystic plate and associated with a variant branching pattern of the parent vessel. J Hepatobiliary Pancreat Sci. 2015; 22(5): 402-403, doi: 10.1002/jhbp.231, indexed in Pubmed: 25755109.

8. El Amrani M, Pruvot FR, Truant S. Management of the right hepatic artery in pancreaticoduodenectomy: a systematic review. J Gastrointest Oncol. 2016; 7(2): 298-305, doi: 10.3978/j.issn.2078-6891.2015.093, indexed in Pubmed: 27034799.

9. Hiatt JR, Gabbay J, Busuttil RW. Surgical anatomy of the hepatic arteries in 1000 cases. Ann Surg. 1994; 220(1): 50-52, doi: 10.1097/00000658-199407000-00008, indexed in Pubmed: 8024358.

10. Kullman $E$, Borch $K$, Lindström $E$, et al. Value of routine intraoperative cholangiography in detecting aberrant bile ducts and bile duct injuries during laparoscopic cholecystectomy. Br J Surg. 1996; 83(2): 171-175, indexed in Pubmed: 8689155.

11. Li X, Zhang X, Lu Q, et al. An accessory right hepatic artery derived from the superior mesenteric artery for anterior right liver lobe supply: a case report. Surg Radiol Anat. 2019; 41(8): 969-971, doi: 10.1007/s00276-018-2173-3, indexed in Pubmed: 30580394.

12. Mugunthan N, Kannan R, Jebakani CF, et al. Variations in the origin and course of right hepatic artery and its surgical significance. J Clin Diagn Res. 2016; 10(9): AC01-AC04, doi: 10.7860/JCDR/2016/22126.8428, indexed in Pubmed: 27790413.

13. Nagral S. Anatomy relevant to cholecystectomy. J Minim Access Surg. 2005; 1(2): 53-58, doi: 10.4103/09729941.16527, indexed in Pubmed: 21206646.
14. Olewnik $\measuredangle$, Wysiadecki G, Polguj M, et al. Types of coeliac trunk branching including accessory hepatic arteries: a new point of view based on cadaveric study. Folia Morphol. 2017; 76(4): 660-667, doi: 10.5603/FM.a2017.0053, indexed in Pubmed: 28612916.

15. Polguj $M$, Podgórski $M$, Hogendorf $P$, et al. Variations of the hepatobiliary vasculature including coexistence of accessory right hepatic artery with unusually arising double cystic arteries: case report and literature review. Anat Sci Int. 2014; 89(3): 195-198, doi: 10.1007/s12565013-0219-5, indexed in Pubmed: 24310410.

16. Strasberg SM. A three-step conceptual roadmap for avoiding bile duct injury in laparoscopic cholecystectomy: an invited perspective review. J Hepatobiliary Pancreat Sci. 2019; 26(4): 123-127, doi: 10.1002/jhbp.616, indexed in Pubmed: 30828991.

17. Strasberg S, Brunt M. Rationale and use of the critical view of safety in laparoscopic cholecystectomy. J Am Coll Surg. 2010; 211(1): 132-138, doi: 10.1016/j.jamcollsurg.2010.02.053.

18. Tsuruda $Y$, Okumura $H$, Setoyama $T$, et al. Laparoscopic cholecystectomy with aberrant bile duct detected by intraoperative fluorescent cholangiography concomitant with angiography: A case report. Int J Surg Case Rep. 2018; 51: 14-16, doi: 10.1016/j.ijscr.2018.08.009, indexed in Pubmed: 30130667.

19. Uy PP, Francisco DM, Trivedi A, et al. Vascular diseases of the spleen: a review. J Clin Transl Hepatol. 2017; 5(2): 152-164, doi: 10.14218/JCTH.2016.00062, indexed in Pubmed: 28660153.

20. Whitley $A$, Oliverius $M$, Kocián $P$, et al. Variations of the celiac trunk investigated by multidetector computed tomography: Systematic review and meta-analysis with clinical correlations. Clin Anat. 2020; 33(8): 1249-1262, doi: 10.1002/ca.23576, indexed in Pubmed: 32012339.

21. Yamaguchi T, Hasegawa K, Sauvain MO, et al. An aberrant right hepatic artery arising from the gastroduodenal artery: a pitfall encountered during pancreaticoduodenectomy. Surg Today. 2021; 51(10): 1577-1582, doi: 10.1007/ s00595-021-02242-4, indexed in Pubmed: 33575949.

22. Zaki SM, Abdelmaksoud AHK, Khaled BEA, et al. Anatomical variations of hepatic artery using the multidetector computed tomography angiography. Folia Morphol. 2020; 79(2): 247-254, doi: 10.5603/FM.a2019.0090, indexed in Pubmed: 31436302. 